\title{
Mejora de la satisfacción corporal en la madurez a través de un programa específico de imagen corporal ${ }^{*}$
}

\section{Improving Body Satisfaction at Maturity through Specific Body Image Programs}

\author{
Roberto Sánchez Cabrero a \\ Universidad Alfonso X El Sabio, España, España \\ ORCID: https://orcid.org/0000-0002-1978-7531
}

a Autor de correspondencia. Correo electrónico: rcabrero@uax.es

Para citar este artículo: Sánchez Cabrero, R. (2020). Mejora de la satisfacción corporal en la madurez a través de un programa específico de imagen corporal. Universitas Psychologica, 19. https://doi.org/10 .11144/Javeriana.upsy19.mscm

\begin{abstract}
RESUMEN
Se compara la mejora lograda en satisfacción corporal por un programa específico de intervención en imagen corporal en personas mayores con los resultados obtenidos en programas de intervención y animación social, con el propósito de valorar si son significativamente mejores que otros programas de carácter social. De ser así, resulta relevante intervenir específicamente sobre la satisfacción corporal durante la vejez para evitar otros problemas relacionados como la depresión o la baja autoestima. En el estudio, se analizan las conexiones encontradas entre el género, la edad y la presencia de pareja sentimental estable, como características de gran importancia para la satisfacción corporal. Los resultados muestran que, aunque la relevancia de la satisfacción corporal durante esa etapa es cualitativamente diferente a edades anteriores, la intervención específica en imagen corporal es significativamente más efectiva que otros programas preventivos o que la maduración experimental.
\end{abstract}

Palabras clave

satisfacción corporal; madurez; vejez; imagen corporal; personas mayores.

\begin{abstract}
The present study compares the improvement achieved by a specific programme about body image versus a social intervention programme for the elderly. The study also analyses the connections found among gender, age and presence or absence of a partner, as features of great significance towards body satisfaction. The objective is to assess whether the specific programmes about body image improve body satisfaction in old age significantly better than social intervention programmes and therefore, whether it is important to improve the intervention on the body satisfaction during the old age to avoid other problems such as depression or low self-esteem. Results show that, although the relevance of body satisfaction during old age is qualitatively different from previous ages, the specific intervention in body image improves participants' body satisfaction significantly more than other preventive programmes or that the experimental maturation.
\end{abstract}

Keywords

body satisfaction; maturity; old age; body image; elderly. 
Tal y como afirman McDermott et al. (2015), puede entenderse de manera amplia el concepto de imagen corporal como la "sensación general que tiene una persona sobre su apariencia física y función corporal" (p. 353). Esta definición se concreta de manera práctica como la forma en que uno siente, percibe, imagina y actúa respecto a su apariencia física (Cash, 2017).

Es posible desglosar la imagen corporal en dos dimensiones cualitativamente distintas. En primer lugar, la dimensión perceptiva, derivada de la evaluación del tamaño y las proporciones del propio cuerpo y, en segundo lugar, la dimensión cognitivo-emocional, también denominada satisfacción corporal, que será la variable medida en esta investigación (Maganto, Garaigordobil, \& Kortabarria, 2017).

Se puede definir la satisfacción corporal como 'el grado de aceptación que tiene una persona a la hora de valorar su aspecto físico' (Benton \& Karazsia, 2015). Esta valoración suele ser categorizada como negativa, cuando disminuye su confianza ante los demás y despierta emociones negativas, y positiva, cuando le da un plus de confianza al afrontar las relaciones sociales (Tylka \& Wood-Barcalow, 2015). Cuando una persona deja atrás su juventud y se adentra en la madurez o en la vejez (considerando los 50 años como punto de corte), las preocupaciones y dificultades asociadas a una imagen corporal negativa suelen deberse exclusivamente a carencias en satisfacción corporal, ya que la distorsión perceptiva asociada a las dimensiones y apariencia de su cuerpo físico, habituales durante la adolescencia y juventud en los trastornos de la imagen corporal, son muy poco frecuentes al llegar a la madurez (Sabik \& Versey, 2016; Sánchez-Cabrero \& Maganto, 2009; Vega, Ramos, Barrios, \& Quintero, 2015).

La razón principal por la cual la distorsión perceptiva que se da habitualmente en los trastornos vinculados a una imagen corporal negativa entre adolescentes y jóvenes, no suele darse cuando una persona llega al momento en el que reconoce en sí misma una madurez física, o percibe su cuerpo como envejecido, es debido a que el epicentro de la preocupación por la imagen corporal se traslada, durante la madurez y la vejez, hacia otros defectos físicos más asociados al deterioro físico derivado de la edad. La literatura científica ha comprobado cómo las principales preocupaciones sobre el aspecto físico ante los demás cuando la persona se siente madura o mayor, son los signos de la edad, es decir, la inquietud se dirige especialmente a la pérdida de condiciones físicas, a las arrugas, a la caída de pelo, al olor corporal y a otros signos tradicionalmente vinculados con la vejez (Gubrium \& Holstein, 2006; Longo, 2015), al contrario que en otras épocas vitales, en las que la principal preocupación por la apariencia está vinculada especialmente con la delgadez (Fernández, González, Contreras, \& Cuevas, 2015; Grogan, 2016; Kvalem, Træen, Markovic, $\&$ von Soest, 2019).

El carácter evolutivo y progresivo de las señales más visibles de la madurez y la senectud favorece que la persona acepte con mayor facilidad la progresiva transformación y deterioro de su aspecto físico. Aunque otras circunstancias, como el alejamiento definitivo del ideal físico social deseado, también favorecen que la satisfacción corporal disminuya y sea más frecuente tener bajas expectativas acerca de la evolución futura de la apariencia física. Estas diferencias justifican que entre las personas maduras y mayores sea muy infrecuente padecer trastornos de la alimentación, y en cambio, sea mucho más frecuente que se den procesos asociados a síntomas depresivos relacionados con la popularmente conocida como "crisis de madurez", que se origina principalmente a través del reconocimiento, en la propia persona en proceso de madurez o vejez, de los signos de la edad vinculados con la senectud (Gubrium \& Holstein, 2006; Zeman \& Zeman, 2015).

A nivel científico, los posibles vínculos entre la percepción de la propia senectud, fruto del deterioro de la su apariencia, y sus implicaciones sobre el estado psicológico y emocional en la persona envejecida resultan de gran interés (Sánchez-Cabrero, Carranza, Novillo, \& Pericacho, 2019), ya que el deterioro del aspecto del propio cuerpo es, sin duda alguna, para una persona en proceso de envejecimiento, 
la señal más inequívoca indicativa de la llegada de la senectud y cercanía de la muerte (Schoufour et al., 2018). Supone que el individuo se vea a sí mismo ejerciendo un rol muy poco valorado y socialmente temido, el del anciano o persona mayor, a modo de autoesquema personal a la hora de afrontar las interacciones sociales en su entorno cercano (Kihlstrom, 2015). Esta autoidentificación del individuo como persona envejecida conlleva una aceptación paulatina de sus nuevos limitantes y circunstancias desfavorecidas, lo que puede generar dificultades e inconvenientes de carácter emocional, como ansiedad, síntomas depresivos o estrés, debido a una deficiente aceptación de sus limitaciones o a la autoidentificación con roles sociales negativos que parten de prejuicios sociales poco recomendables hacia la senectud, pero muy frecuentes (Bratt, Abrams, Swift, Vauclair, \& Marques, 2018). La intervención terapéutica sobre la imagen corporal de personas maduras o mayores puede contribuir a que logren aceptarse a sí mismas adecuadamente y a desarrollar un "yo" positivo al llegar a la madurez y a la ulterior senectud (Hudson, Lucas, \& Donnellan, 2016; Kozar, 2004; Mangweth-Matzek et al., 2006; McLean, Paxton, \& Wertheim, 2011; Mellor, Connaughton, McCabe, \& Tatangelo, 2017). Cobra una gran relevancia, pues, desarrollar nuevas investigaciones en este campo para aportar luz sobre cuál es el importante papel que juega la imagen corporal en la sociedad de hoy, y qué relevancia tienen las expectativas acerca del aspecto físico ante los demás, en todo este proceso de adaptación a las últimas fases de la vida.

Existe amplio consenso científico respecto a la consideración de la satisfacción corporal y la imagen corporal como indicadores claramente mejorables mediante la participación en programas de intervención social estructurados, que incidan en la interacción social positiva (Cash, 2017; Voelker, Reel, \& Greenleaf, 2015), desde los programas de intervención clásicos de Cash (1997) y el Programa preventivo sobre imagen corporal y trastornos de la alimentación (PICTA) de Maganto, del Río y Roiz (2002), hasta los actuales como los de Rosés-Gómez
(2014), Kilpela et al. (2016), Halliwell et al. (2016), McCabe, Connaughton, Tatangelo, Mellor y Busija (2017) o Bailey, Gammage y Van Ingen (2019). Sin embargo, ninguno de ellos está destinado a personas mayores, centrándose, en cambio, en adolescentes o jóvenes, y principalmente en la población femenina, excepto el Programa de mejora de la autoestima y la imagen corporal para adultos (IMAGINA) de Sánchez-Cabrero (2012), por lo que es necesario profundizar en la acomodación de los beneficios de estos programas sobre este colectivo.

En el caso específico de España, investigar sobre la madurez y vejez se hace incluso más importante, puesto que hoy por hoy es uno de los países más envejecidos del mundo (Instituto Nacional de estadística [INE], 2017), y la tendencia actual indica que irá en aumento en los próximos años, convirtiéndose en una de las preocupaciones sociales más importantes para el análisis psicosocial, en los años venideros (Abellán, Ayala, \& Pujol, 2017).

En el presente estudio, se toma como punto de corte la edad de 50 años, como la más aceptada socialmente como inicio de la madurez personal. Se analiza el factor edad en seis agrupaciones de cinco años cada una, salvo la última, obviamente abierta, para observar posibles cambios asociados a la edad respecto a la satisfacción corporal. Además de la edad, como factor de relevancia para la satisfacción corporal, existe otra serie de factores relevantes que se incluyen en este estudio para su análisis: el sexo y la presencia o ausencia de pareja.

Por lo que respecta al sexo como variable interviniente, este se sigue considerando como un factor de gran incidencia sobre la imagen corporal al llegar a la madurez, tal y como muestran investigaciones recientes (Cundall \& Guo, 2017; Homan \& Tylka, 2018; Hudson et al., 2016; Tylka \& Homan, 2015), en las que se pone de manifiesto que las mujeres siguen padeciendo, por lo general, menor satisfacción corporal a partir de la madurez, al igual que en el resto de edades. No obstante, existe poca información sobre la mejora de la satisfacción corporal según 
sexo con programas destinados a ello, debido a la falta de investigaciones en este aspecto.

Otra variable que puede tener gran incidencia sobre la imagen corporal en la madurez y la vejez es la presencia o ausencia de pareja estable. Hay investigaciones que reflejan evidencias claras de que ambas variables están estrechamente vinculadas (Markey, Markey, \& Birch, 2005; Rodgers, McLean, \& Paxton, 2015), aunque algunos autores afirman que la presencia de pareja no siempre tiene efectos beneficiosos para la satisfacción corporal, y podría tener una influencia invertida según sexos que enmascara su influencia real (Sánchez-Cabrero, Carranza, Novillo, \& Pericacho, 2019).

El carácter cognitivo-emocional de los problemas vinculados a una imagen corporal negativa durante la tercera edad, y en concreto con una baja satisfacción corporal, hace que este colectivo se beneficie enormemente en estos aspectos del aumento de la participación social. Está demostrado que tener una vida social activa y una amplia red social de contactos frecuentes se asocia directamente con una imagen corporal más positiva durante esa etapa. Por lo tanto, la concurrencia a programas de participación e intervención social mejora la satisfacción corporal, aunque es difícil saber hasta qué punto, debido a la carencia de investigaciones al respecto. También falta concretar la influencia de programas específicamente destinados a mejorar la imagen corporal, que estén, además, específicamente destinados a la población mayor (Mellor et al., 2017).

Para dar respuesta a todos los interrogantes planteados, este proyecto de investigación se plantea responder a dos objetivos principalmente: 1) comparar la mejora producida por un programa de intervención específico en imagen corporal aplicado sobre la satisfacción corporal de sus participantes frente a tratamientos de control con circunstancias similares, y 2) valorar las relaciones existentes entre las variables incluidas en el estudio (sexo, edad y presencia o ausencia de pareja), como variables de gran influencia sobre la imagen corporal. Responder a ambos objetivos puede servir para establecer la importancia de desarrollar programas de intervención en imagen corporal al llegar a la madurez y a la senectud, con vistas a mejorar la intervención sobre las personas en proceso de vejez con incipientes problemas emocionales, tales como el estado de ánimo deprimido o la baja autoestima, inconvenientes frecuentemente asociados a la vejez (Abellán et al., 2017).

\section{Método}

\section{Muestra}

La muestra fue seleccionada a través de su participación en distintos programas de intervención social de la Cruz Roja Española, utilizándose para la obtención de los sujetos experimentales un muestreo por conglomerados, en 10 localidades de la provincia de Zamora, España.

Un total de 176 participantes mayores de 50 años $(M=64.03$ años, $D E=8.06)$ participaron en este estudio: 83 de los cuales eran menores de 65 años y 93 con edades superiores a los 65 años. La distribución por sexos fue de 30 hombres y 146 mujeres. La distribución de los participantes a las condiciones experimentales fue mediante muestreo aleatorio, garantizándose así el diseño experimental.

\section{Instrumentos}

Como variable independiente para el estudio se utilizó un programa específico de intervención en imagen corporal. En concreto, el Programa de mejora de la autoestima y la imagen corporal para adultos ([IMAGINA]; Sánchez-Cabrero, 2012), por estar destinado para personas mayores de 50 años y ser un instrumento único en su categoría.

Consta de ocho sesiones de 90-120 minutos de aplicación colectiva. Las actividades tienen un carácter lúdico y se basan en la interacción social positiva. Tienen como objetivo mejorar la imagen corporal y la autoestima de los participantes, fomentar la participación social, la aceptación de la apariencia física y una nutrición saludable. 
También promueven la inteligencia emocional, la interacción social positiva y reevalúan las expectativas personales relacionadas con la apariencia física y la interacción social, que pueden ser perjudiciales para la salud mental del participante. Este programa es único en su campo y, por lo tanto, es la mejor opción para estudiar la mejora de la imagen corporal de los participantes, en este estudio. En la encuesta de satisfacción de los participantes a su prueba piloto, obtuvo una excelente aceptación (9 de 10) (SánchezCabrero, 2012).

Como control, se utilizaron programas de animación e intervención social de la Cruz Roja Española para personas mayores, con la misma duración y también de aplicación colectiva. Estos programas también fomentan la interacción social positiva y tienen un carácter lúdico. La principal diferencia estriba en la atención por la imagen corporal y, en concreto, por la satisfacción corporal.

Para la evaluación de satisfacción corporal como variable dependiente (VD), se utilizó el Body Shape Questionnaire ([BSQ]; Cooper, Taylor, Cooper, \& Fairburn, 1987), adaptado al español y validado sobre población española por Raich et al. (1996). A pesar de su antigüedad, sigue utilizándose en el ámbito científico y es la herramienta más aceptada y válida en la actualidad (Baile, Guillén, \& Garrido, 2002; Fernández et al., 2015). El test BSQ cuenta con una elevada confiabilidad interna ( $\alpha$ de Cronbach entre 0.95 y 0.97 ) y validez concurrente con otros instrumentos similares como el Multidimensional Body Self-Relations Questionnaire ([MBSRQ]; Cash, 2015) y la subescala de insatisfacción corporal del Eating Disorders Inventory ([EDI]; Garner, Olmstead, \& Polivy, 1983). Este instrumento fue seleccionado por su sencillez de aplicación a población de adultos mayores, ya que tan solo consta de 34 ítems, y presenta un léxico sencillo. El rango máximo de puntuaciones oscila entre 34 y 170, asociándose las menores puntuaciones, de manera inversa, a mayor satisfacción corporal.

\section{Diseño y procedimiento}

Se parte de un diseño experimental bivariado, con una variable independiente (VI inter) o tratamiento (programa específico sobre satisfacción corporal frente a un programa de intervención social) y una variable dependiente (satisfacción corporal), utilizando para el contraste la comparación entre grupos, experimental y control, y las medidas pre y post tratamiento (VI intra), dando lugar a un diseño mixto inter-intra.

Se controlaron distintas variables de bloqueo a través de la selección de la muestra y la aleatorización de los sujetos a las condiciones experimentales. Se valoraron, además, la influencia e interacción del sexo, edad y la presencia o ausencia de pareja actual como variables intervinientes en el estudio.

La muestra se dividió en 10 grupos distintos para la aplicación de los tratamientos (cinco para el programa específico sobre imagen corporal y cinco para los programas de mediación e intervención social). El grupo experimental participó en el IMAGINA (Sánchez-Cabrero, 2012), durante ocho sesiones grupales de dos horas cada una (dos veces por semana, sumando un total de cuatro semanas). El grupo de control participó en un programa psicosocial no específico, implementado por la Cruz Roja Española, también con ocho sesiones de dos horas de duración cada una.

El BSQ se aplicó en dos momentos diferentes. Es decir, antes y después del tratamiento experimental. El pretest con los grupos ya formados y antes de participar en su programa asignado; el postest inmediatamente después del final de la última sesión del programa. La aplicación de BSQ se realizó en papel y de forma individual, aunque todos los participantes de cada grupo se encontraban en la misma sala al mismo tiempo. Cada medición tuvo una duración aproximada de diez minutos para responder a 34 ítems.

Se empleó el consentimiento escrito informado de la muestra para el estudio, garantizando en todo momento sus derechos 
según los principios recomendados en la Declaración de Helsinki (World Medical Association [WMA], 2013), y archivando correctamente los datos de investigación en un servidor seguro.

Los datos recogidos en el estudio fueron analizados estadísticamente, teniendo en cuenta las necesidades de un diseño experimental de medidas repetidas con diferenciación de grupo experimental y de control. La presencia de grupo de control, de manipulación de variable independiente (VI) y de asignación aleatoria de los sujetos experimentales a cualquiera de ambas condiciones (experimental y control) garantiza el diseño experimental. El hecho de que sea un estudio inter-intra ofrece, además, gran robustez a los resultados obtenidos.

Se emplearon dos tipos de pruebas estadísticas inferenciales para analizar los resultados. En primer lugar, el contraste de diferencia de medias para muestras emparejadas, utilizando la $t$ de Student como estadístico de contraste. El resultado de esta prueba sirve para comparar la medición pre de la variable dependiente con la medición post, tanto en la condición experimental como de control. En segundo lugar, para comparar ambas condiciones experimentales, cuando al menos una de ellas es una variable intrasujeto de medidas repetidas, es recomendable realizar contrastes multivariados a través del ANOVA de medidas repetidas, utilizando la Traza de Pillai y la Lambda de Wilks como estadísticos de contraste. Este análisis permite evaluar la combinación de variables inter e intrasujeto y valorar su significación.

\section{Resultados}

Para responder al primer objetivo de investigación, en la Tabla 1, se muestran las medias aritméticas y desviaciones estándar de las distintas mediciones realizadas en la evaluación pre-tratamiento y post-tratamiento con el test BSQ y la $t$ de Student y su significación estadística sobre la diferencia de medias prepost según las condiciones experimentales (experimental y de control).

\section{Tabla 1}

Medias y desviaciones típicas del test BSQ según la condición experimental en las fases pretest, postest y en la diferencia pretest - postest

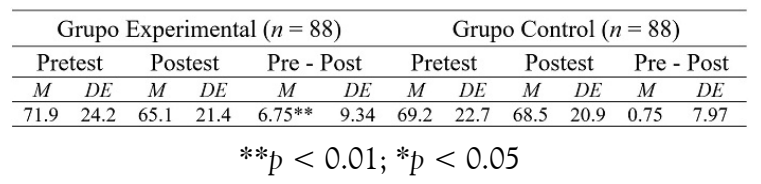

La Tabla 1 muestra con claridad cómo la mejora debida al programa específico aplicado de intervención en imagen corporal (IMAGINA) logra en sus participantes considerablemente mayor mejora en satisfacción corporal (medido con el test BSQ) que los programas de control (6.75 frente a 0.75). La diferencia entre la medición pre $\mathrm{y}$ post es significativa $(\alpha=$ 0.01) según el contraste de diferencia de medias $t$ de Student, solamente en la condición experimental, lo que es indicador de que la condición experimental ofrece una considerable mejora en satisfacción corporal respecto a la condición de control. No obstante, este resultado debe confirmarse como significativo mediante contrastes multivariados con el ANOVA de medidas repetidas, donde se evalúa el grado de significación estadística de los efectos de la variable "condición experimental" (VI inter) sobre la mejora en satisfacción corporal de los sujetos experimentales (VI intra), tal como puede verse en la Tabla 2.

\section{Tabla 2}

Contrastes multivariados de los efectos de la variable "Condición" a través del ANOVA de Medidas repetidas

\begin{tabular}{|c|c|c|c|c|c|}
\hline Efecto & Estadísticos & Valor & $F$ & $g /$ hip. & $g l$ error \\
\hline \multirow{2}{*}{$\begin{array}{l}\text { Diferencia en la medida PRE y } \\
\text { POST tratamiento del test BSQ } \\
\text { (Intra) }\end{array}$} & Traza de Pillai & 0.16 & $32.84^{* * *}$ & 1 & 174 \\
\hline & Lambda de Wilks & 0.84 & $32.84 * *$ & 1 & 174 \\
\hline $\begin{array}{l}\text { Relación de la variable } \\
\text { Condición (inter) con la }\end{array}$ & Traza de Pillai & 0.11 & $21.02 * *$ & 1 & 174 \\
\hline $\begin{array}{l}\text { medición PRE y POST } \\
\text { tratamiento del test BSQ (Intra) }\end{array}$ & Lambda de Wilks & 0.89 & $21.02 * *$ & 1 & 174 \\
\hline
\end{tabular}

La Tabla 2 confirma que el programa específico aplicado sobre imagen corporal (VI inter) desarrolla una mejora en satisfacción corporal (VI intra) significativamente superior a la desarrollada mediante los programas de control, ya que las diferencias observadas entre la condición experimental y de control en la 
mejora en satisfacción corporal (diferencia prepost) es significativa en todos los estadísticos considerados $(\alpha=0.01)$.

En respuesta al segundo objetivo de investigación, en la Tabla 3, se muestran las medias aritméticas y desviaciones estándar de las distintas mediciones realizadas en la evaluación pre-tratamiento y post-tratamiento y la $t$ de Student y su significación estadística de la diferencia de medias pre-post, según las condiciones experimentales (experimental y control) y según todas las variables intervinientes consideradas en el estudio.

\section{Tabla 3}

Medias y desviaciones típicas del test BSQ según variables intervinientes en las fases pretest, postest y en la diferencia pretest-postest

\begin{tabular}{|c|c|c|c|c|c|c|}
\hline \multirow{3}{*}{ Variables } & \multicolumn{3}{|c|}{ Grupo Experimental $(n=88)$} & \multicolumn{3}{|c|}{ Grupo Control $(n=88)$} \\
\hline & Pretest & Postest & Pre-Post & Pretest & Postest & Pre - Post \\
\hline & \multicolumn{3}{|c|}{ Sexo } & $M(D E)$ & $M(D E)$ & $M(D E)$ \\
\hline $\begin{array}{l}\text { Mujer }(n=146) \\
\text { Hombre }(n=30)\end{array}$ & $71.9(25.2)$ & $65.3(22)$ & $6.6^{* * *}(9.85)$ & $72.3(21.4)$ & $71.3(19.8)$ & $1.07(8.29)$ \\
\hline \multirow{2}{*}{\multicolumn{7}{|c|}{ Edad }} \\
\hline & & & & & & \\
\hline $\begin{array}{l}\text { De } 50 \text { a } 54(n=30) \\
\text { De } 55 \text { a } 59(n=22)\end{array}$ & $\begin{array}{l}78.3(28.6) \\
87.9(25.1)\end{array}$ & $\begin{array}{r}66.6(23.3) \\
80.1(2.9)\end{array}$ & $\begin{array}{r}11.74 * *(10.89) \\
7.71(10.13)\end{array}$ & $\begin{array}{l}80.1(25.9) \\
72.9(20.9)\end{array}$ & $\begin{array}{r}78(23) \\
73.3(23.2)\end{array}$ & $\begin{array}{r}2.09(6.11) \\
-0.33(6.33)\end{array}$ \\
\hline De 60 a $64(n=31)$ & $72.8(23.1)$ & $68.5(19.5)$ & $4.31^{*}(7.52)$ & $75(25.5)$ & $76.3(20.7)$ & $-1.33(8.72)$ \\
\hline  & $72.1(24.8)$ & $65.5(24.5)$ & $6.55^{* *}(7.92)$ & $72(20.5)$ & $69.2(17.9)$ & $2.72(9.51)$ \\
\hline De 70 a $74(n=27)$ & $64.9(15.8)$ & $59.3(15.1)$ & $5.64(10.29)$ & $57.6(18.6)$ & $58.4(15.4)$ & $-0.77(8.16)$ \\
\hline $75 \mathrm{o}+(n=19)$ & $56.1(16.6)$ & $53.6(16.7)$ & $2.5(7.62)$ & $49.2(12.4)$ & $48.1(12.1)$ & $1.11(6.41)$ \\
\hline \multicolumn{7}{|c|}{ e pareja estable } \\
\hline$=117)$ & $73.8(23.4)$ & $67.3(19.9)$ & $6.53 * *(10.12)$ & $71.3(23.7)$ & $69.9(21.8)$ & $1.35(8.23)$ \\
\hline Separa & $104.7(2-r) h a$ & $92(2$ & 12.67 & $89.7(15.9)$ & & $1.5(4.43)$ \\
\hline & 63.2 & $56.2(16.7)$ & $7^{* * *}(6.51)$ & $49(10)$ & $52.8(12.3)$ & $-3.8(5.36)$ \\
\hline Viudez & 65.1 & $58.8(24.1)$ & $6.75^{* * *}(9.34)$ & $63.6(18.3)$ & $63.7(16.8)$ & $-0.11(8.24)$ \\
\hline
\end{tabular}

Es perceptible en la Tabla 3 cómo todas las condiciones de todas las variables intervinientes consideradas muestran mayor mejora en satisfacción corporal en la condición experimental que en la condición de control, resultando, en la mayoría de casos, en diferencias significativas, todas para la condición experimental y ninguna para la condición de control. Este resultado obtenido refleja, en primer lugar, que el efecto de la condición experimental sobre la satisfacción corporal es mayor que cualquiera del resto de variables evaluadas (sexo, edad y presencia de pareja estable), puesto que ninguna de ellas logra difuminar el efecto de la condición experimental que puede verse en la Tabla 1.

En relación con la variable sexo, los contrastes $t$ de Student son significativos $(\alpha=0.01)$ para la diferencia de medias pre-post en la condición experimental, mientras que en la condición control la diferencia observada no es significativa. En el programa específico sobre imagen corporal aplicado, se puede observar una mayor mejora de los hombres en comparación con las mujeres en satisfacción corporal (en mujeres 6.6 y en hombres 7.57); en cambio, la participación de los hombres en los programas de control muestra una ligera tendencia a que podría generar un empeoramiento en su satisfacción corporal, puesto que las puntuaciones de las mujeres son positivas, aunque bajas (1.07), y las de los hombres son negativas (-0.69). Sin embargo, esta tendencia no se muestra significativa, por lo que es posible que este resultado se deba a que el aumento de la interacción social en los hombres los hace más conscientes de su imagen corporal, incrementando sensiblemente su preocupación por la apariencia física ante los demás, efecto que se camufla si el programa es específico.

Respecto a la variable edad, los contrastes $t$ de Student son significativos $(\alpha=0.01)$ para la diferencia de medias pre-post en la condición experimental, en el caso del rango de edad de 50 a 54 años (11.74) y en el rango de edad de 65 a 69 años (6.55). También son significativos para la condición experimental $(\alpha=0.05)$ en el rango de edad de 60 a 64 años (4.31), por lo que puede afirmarse que el programa específico sobre satisfacción corporal aplicado provoca mejoras estadísticamente significativas en estos rangos de edades. En lo que respecta a la condición de control, en cambio, no se obtiene ninguna medición significativa.

Respecto a la variable presencia o ausencia de pareja estable, los contrastes $t$ de Student son significativos $(\alpha=0.01)$ para la diferencia de medias pre-post en la condición experimental en los grupos de participantes con pareja estable (6.53), para los participantes viudos (6.75) y en la condición de soltería (7) y en el grupo de participantes separados o divorciados (12.67), considerando un nivel de confianza del $95 \%(\alpha=$ 0.05). Para los grupos de control, ninguna medida es significativa, por lo que puede afirmarse que no hay condiciones de presencia de pareja estable que sean significativas en programas de control.

Estos resultados deben interpretarse con moderación, puesto que la escasez de 
participantes en las condiciones de algunas variables puede sesgar fácilmente los análisis estadísticos. A modo de muestra, una evaluación superficial de los resultados puede llevar a concluir que para los individuos separados, el programa específico aplicado sobre satisfacción corporal no provoca mejoras significativas. Sin embargo, los sujetos experimentales separados o divorciados son muy pocos $(N=7)$, siendo asignados cuatro participantes a la condición de control y tres a la condición experimental, cifras claramente insuficientes como para extrapolar estos resultados a la población de referencia. También deben ser tomados con moderación los resultados de individuos solteros, ya que $N$ en esta condición solamente asciende a 15 participantes, por lo que el empeoramiento en su satisfacción corporal (-3.8) fruto de su participación en programas de control, además de no ser significativo, son datos tomados sobre escasa muestra. Tomando como referencia las condiciones de la variable Presencia actual de pareja estable en las que se da una muestra $(\mathrm{N})$ amplia (con pareja estable y viudez), la diferencia de medias de la condición experimental resultan estadísticamente significativas, lo que indica que el programa específico aplicado sobre imagen corporal provoca mejoras significativas en satisfacción corporal, independientemente de si el participantes tiene o no pareja sentimental estable, siempre y cuando la condición evaluada cuente con muestra suficiente.

En los resultados, se puede apreciar cómo los participantes separados o divorciados son los que muestran globalmente una menor satisfacción corporal en la condición pre $(\mathrm{BSQ}=96.1)$, lo que parece indicar que su estado civil influye negativamente sobre su satisfacción corporal. Los participantes separados o divorciados, por otro lado, muestran la mayor mejora obtenida en el estudio (12.67). No obstante, estos resultados deben tomarse con moderación, puesto que la muestra en esta condición es muy escasa $(N=7)$. En relación con los participantes solteros, estos son los que muestran una mayor satisfacción corporal (BSQ . 58.5) y, por lo que respecta a los participantes viudos, teniendo en cuenta también que son mayoritariamente mayores, se repite en gran medida lo ya visto en el análisis de la variable edad, por lo que ambas condiciones muestran resultados muy relacionados y se puede considerar, complementarios. Una conclusión global derivada de estas diferencias, según la presencia de pareja estable, es que puede afirmarse que se trata de una variable de gran relevancia para la satisfacción corporal, ya que existen notables diferencias en la valoración pretratamiento. En cambio, la relevancia de este factor no muestra una significación mayor para la mejora debido al programa específico aplicado sobre imagen corporal.

Las diferencias observadas en los distintos contrastes $t$ de Student en el programa específico aplicado sobre imagen corporal, según las distintas variables intervinientes medidas, deben ser analizadas para evaluar su significación estadística a través de los contrastes multivariados mediante el análisis del ANOVA de Medidas repetidas, cuyos resultados se muestran en la Tabla 4.

\section{Tabla 4}

Contrastes multivariados de las variables intervinientes sexo y edad sobre las medidas tomadas teniendo en cuenta la variable "Condición"

\begin{tabular}{|c|c|c|c|c|c|c|}
\hline Efecto & Estadisticos & Valor & $F$ & $\begin{array}{c}g l \\
\text { hip. }\end{array}$ & $\begin{array}{c}g l \\
\text { error }\end{array}$ & $p$ \\
\hline \multirow{2}{*}{$\begin{array}{l}\text { Relación de la variable Sexo (inter) } \\
\text { con la medición Pre y Post } \\
\text { tratamiento del test BSQ (intra) }\end{array}$} & Traza de Pillai & 0 & 0.05 & 1 & 172 & 0.82 \\
\hline & Lambda de Wilks & 1 & 0.05 & 1 & 172 & 0.82 \\
\hline \multirow{2}{*}{$\begin{array}{l}\text { Relación de la variable Sexo (inter) } \\
\text { con la medición Pre y Post } \\
\text { tratamiento del test BSQ (intra) } \\
\text { teniendo en cuenta la variable } \\
\text { Condición (inter) }\end{array}$} & Traza de Pillai & 0 & 0.61 & 1 & 172 & 0.44 \\
\hline & Lambda de Wilks & 1 & 0.61 & 1 & 172 & 0.44 \\
\hline \multirow{2}{*}{$\begin{array}{l}\text { Relación de la variable Edad } \\
\text { (inter) con la medición Pre y Post } \\
\text { tratamiento del test BSQ (intra) }\end{array}$} & Traza de Pillai & 0.05 & 1.6 & 5 & 164 & 0.16 \\
\hline & Lambda de Wilks & 0.95 & 1.6 & 5 & 164 & 0.16 \\
\hline \multirow{2}{*}{$\begin{array}{l}\text { Relación de la variable Edad } \\
\text { (inter) con la medición Pre y Post } \\
\text { tratamiento del test BSQ (intra) } \\
\text { teniendo en cuenta la variable } \\
\text { Condición (inter) }\end{array}$} & Traza de Pillai & 0.02 & 0.72 & 5 & 164 & 0.61 \\
\hline & Lambda de Wilks & 0.98 & 0.72 & 5 & 164 & 0.61 \\
\hline \multirow{2}{*}{$\begin{array}{l}\text { Relación de la variable presencia } \\
\text { de pareja (inter) con la medición } \\
\text { Pre y Post tratamiento del test BSQ } \\
\text { (intra) }\end{array}$} & Traza de Pillai & 0.01 & 0.68 & 3 & 168 & 0.56 \\
\hline & Lambda de Wilks & 0.99 & 0.68 & 3 & 168 & 0.56 \\
\hline \multirow{2}{*}{$\begin{array}{l}\text { Relación de la variable Presencia } \\
\text { de pareja (inter) con la medición } \\
\text { Pre y Post tratamiento del test BSQ } \\
\text { (intra) teniendo en cuenta la } \\
\text { variable Condición (inter) }\end{array}$} & Traza de Pillai & 0.01 & 0.62 & 3 & 168 & 0.6 \\
\hline & Lambda de Wilks & 0.99 & 0.62 & 3 & 168 & 0.6 \\
\hline
\end{tabular}

La Tabla 4 muestra con claridad cómo ninguno de los contrastes multivariados analizados según todas las variables intervinientes consideradas en esta investigación son significativos en ningún estadístico para ninguna variable o condición, tanto para la mejora debida al 
tratamiento (intra), como si esta se combina con la variable independiente (VI) condición experimental (inter). Estos resultados confirman que el programa específico aplicado sobre imagen corporal genera mejoras significativas para la satisfacción corporal entre sus participantes, independientemente de cualquiera de las condiciones contempladas en cualquiera de las variables evaluadas en esta investigación.

\section{Discusión}

En primer lugar, como conclusión principal de este estudio, en relación con la variable independiente (VI inter) evaluada, es decir, en relación con el efecto del programa de mejora de la imagen corporal IMAGINA (SánchezCabrero, 2012), frente al efecto de tratamientos de control no específicos (programas de animación e intervención social de la Cruz Roja Española para personas mayores), puede afirmarse con rotundidad que, tal y como predice la Teoría de la Imagen Corporal Positiva (Homan \& Tylka, 2018; Tylka \& Homan, 2015), la mejora en satisfacción corporal es significativamente mayor si la intervención se realiza mediante el programa específico de imagen corporal IMAGINA. En el test BSQ, se produce una mejora de 6.75 puntos de media en los participantes al programa específico aplicado sobre imagen corporal, por solo 0.75 en la condición de control. Esta mejora es clara, por lo que se puede concluir que el programa específico aplicado sobre imagen corporal incrementa la satisfacción corporal de los participantes significativamente más que otros programas preventivos inespecíficos o que la propia maduración experimental (Bailey et al., 2019; Rosés-Gómez, 2014; Halliwell et al., 2016).

Estos resultados generales tienen implicaciones clínicas y científicas evidentes, puesto que reflejan con claridad cómo la imagen corporal se puede intervenir de forma efectiva también a partir de la madurez, solventando una tradicional laguna en la literatura científica (Bratt et al., 2018; Hudson et al., 2016; Mellor et al., 2017). Además, también reflejan las bondades y buen diseño del programa seleccionado, IMAGINA de Sanchez-Cabrero (2012), para desarrollar de forma eficiente su labor con muestras de personas en proceso de madurez y mayores.

Teniendo en cuenta que los resultados obtenidos muestran que la preocupación por la apariencia física sigue siendo una preocupación relevante en las últimas fases de la vida, se puede considerar que la inclusión complementaria de la intervención específica sobre la imagen corporal de las personas maduras y mayores puede contribuir significativamente a la atención prestada a pacientes de esas edades que estén inmersos en procesos depresivos o estados de ánimo alterados (Vega et al., 2015; Zeman \& Zeman, 2015).

A partir de estos resultados, se abre una prospectiva más que posible que se dirija a profundizar, por un lado, en los beneficios de la intervención sobre la imagen corporal en personas maduras o mayores y sus posibles beneficios sobre la salud mental de forma preventiva o paliativa. Y, por otro lado, también sería recomendable profundizar científicamente en el proceso de la imagen corporal en la vejez, determinando con claridad sus condicionantes, limitantes y efectos positivos en cuestiones sociales y sobre el estado de ánimo.

Son escasas las investigaciones previas que evalúen la satisfacción corporal en personas en proceso de vejez, por lo que son pocas las referencias con las cuales establecer comparaciones de los resultados de esta investigación. El primero de los estudios con los que puede hacerse una comparación es el de Cooper et al. (1987), en la validación original de BSQ. En su estudio, obtuvo una puntuación media en mujeres de origen anglosajón de 84.7 con una desviación estándar de 28.4. Posteriormente, la adaptación española del test BSQ de Raich et al. (1996) mostró una media de 84.75 y una desviación estándar de 30.42 entre estudiantes universitarias españolas. Ya en el siglo XXI, Baile et al. (2002), con chicas adolescentes españolas, obtuvo media de 81.2 y desviación estándar de 30.25 para chicas de 15-16 años y media de 79.49 y desviación estándar de 31.61 
para chicas de 17-19 años. Otra investigación de actualidad, de Conti, Cordás y Latorre (2009), obtuvo una media de 73.9 entre los adolescentes brasileños, 88.3 para las mujeres y 57.1 para los hombres, lo que refleja una diferencia de género muy pronunciada. Por último, el más reciente estudio de Fernández et al. (2015), afirma haber obtenido resultados muy similares en más de 500 adolescentes y preadolescentes, aunque no muestra medias y desviaciones típicas concretas. Los resultados de este estudio con personas en proceso de madurez y mayores describen una media de 70.54 y desviación estándar de 23.44, por lo que es observable cómo, aunque existen diferencias claras entre las distintas investigaciones y colectivos con edades y circunstancias diferentes, los rangos que incluyen el $68 \%$ de los datos (rango que agrupa el área en torno a una desviación estándar sobre la media) coinciden en más del $60 \%$ de los casos. Por lo tanto, una gran proporción de población en proceso de madurez o vejez muestra niveles similares de insatisfacción corporal que los grupos de adolescentes y jóvenes de los estudios previos.

Por lo que respecta a la variable edad, este estudio ha mostrado cómo, a pesar de que sigue siendo un factor de gran relevancia sobre la satisfacción corporal (es observable una disminución de la preocupación por la apariencia física en edades muy avanzadas), su influencia no impide que haya personas mayores altamente preocupadas por su aspecto físico que se puedan beneficiar con una intervención sobre su imagen corporal, a través de su participación en un programa específico de aplicación colectiva. Los resultados de la evaluación del programa específico aplicado sobre imagen corporal confirman los resultados obtenidos en otras investigaciones, como la investigación de Mangweth-Matzek et al. (2006) con más de 1000 mujeres entre 60 y 70 años, o el longitudinal de McLean et al. (2011), que concluyeron que las preocupaciones sobre el aspecto físico ante los demás no desaparecen al llegar a la vejez. Los resultados obtenidos en este estudio también se muestran coincidentes con otros trabajos previos, como el de Kozar (2004) sobre la incidencia de la publicidad en la vejez, en el que matizan que la edad es una variable relevante, siempre y cuando se combine con otros factores de carácter social, como la percepción de un prolongado deterioro físico o la pérdida de expectativas sociales. En el ámbito científico, estos resultados significan que los programas como IMAGINA tienen gran valor clínico, social y terapéutico en todas las edades, lo que a largo plazo puede derivar en programas estructurados para prevenir o resolver preocupaciones y dificultades de carácter emocional en la imagen corporal en personas mayores.

La teoría de "la vejez como máscara" (Gubrium \& Holstein, 2006) ofrece una explicación plausible a que la imagen corporal siga siendo un factor de preocupación importante para algunas personas en proceso de madurez o vejez, a pesar de que la presión social hacia la delgadez es considerablemente menor que en otras edades. Según esta teoría, las personas en proceso de vejez suelen percibir su aspecto físico como una máscara sobre su auténtico "yo físico", que lo identifican en la imagen que tuvieron de juventud que se refleja en sus fotos. Esta no identificación con el "yo físico actual" en la senectud, podría alterar la satisfacción corporal de forma severa e intensa, tal y como ocurre en la juventud con la presión hacia la delgadez.

Estos resultados apoyan la afirmación realizada por McLean et al. (2011) en su investigación, donde concluyó que la edad no era el factor determinante para que se produzca una reducción considerable en la preocupación por la apariencia, al llegar a la edad madura y a la senectud. Según, los autores para que un individuo reduzca considerablemente la preocupación por el aspecto físico ante los demás, debe observar una gran diferencia con el ideal corporal que se ha marcado, percibir un paulatino distanciamiento con ese ideal y considerar la imposibilidad de acercarse a él en un futuro próximo. Adicionalmente, afirman que la alta correlación de la variable edad con estos requisitos suele provocar confusión, tan común en los estudios científicos que relacionan edad y preocupación por la apariencia. 
En relación con la variable sexo, se puede argumentar, con base en la amplia literatura científica al respecto (Cash, 2017; Grogan, 2016; Rodgers et al., 2015), que una de las principales causas de la diferencia en satisfacción corporal entre hombres y mujeres puede tener su origen en la diferente presión social y percepción de la evaluación social que sienten los hombres en la sociedad occidental actual. No obstante, los resultados obtenidos teniendo en cuenta la variable sexo deben valorarse con sumo cuidado, puesto que la diferencia de muestra entre mujeres $(N=146)$ y hombres $(N=30)$ es considerable y puede sesgar los resultados. Esta situación indeseada se ha debido al mayor interés que muestran las mujeres por la participación en programas sociales de aplicación colectiva respecto a los hombres. Teniendo en cuenta que la participación voluntaria era deseada y la mortalidad experimental entre participantes que pudieran no completar el programa era un riesgo grande, al exigirse ocho sesiones durante cuatro semanas, esta disparidad entre sexos debe aceptarse, ya que refleja esta determinada realidad social.

En lo referente a la presencia de pareja sentimental estable, tradicionalmente se consideraba que los individuos con pareja estable tenían menor preocupación por el aspecto físico ante los demás y parecía lógico considerar que la mayor preocupación podrían tenerla los individuos solteros, ya que la principal función social de la belleza física podría ser la obtención de una pareja adecuada en la juventud. No obstante, los resultados que se obtienen en este estudio parecen indicar que esta consideración no es válida actualmente en personas en proceso de madurez o vejez, ya que son los solteros los que menor preocupación corporal muestran en este estudio (puntuación media de 58.5 en BSQ), y los participantes con pareja estable siguen teniendo una preocupación por la apariencia física considerable, puesto que son, tras los participantes separados o divorciados, los que mayor preocupación corporal muestran. En apoyo a esta conclusión sobre el cambio en valores sociales, Markey et al. (2005) mostraron cómo la insatisfacción corporal de parejas heterosexuales estables no correlacionaba positivamente, y que las mujeres más insatisfechas con su apariencia tendían a distorsionar más la opinión que creían que sus maridos tenían de ellas, independientemente de la opinión real de este. Según los investigadores, el matrimonio y la formación de una familia han perdido importancia como meta social, seguramente debido a los importantes cambios sociales que se han producido en los últimos años, como la igualdad de sexos y la incorporación laboral de la mujer. Estas circunstancias han tenido reflejo en un indicador de preocupación social tan importante como lo es la satisfacción corporal. Por otra parte, una última conclusión interesante sobre la relevancia de la variable interviniente presencia actual de pareja estable es que la mayor mejora debida al programa específico aplicado sobre imagen corporal se da en individuos separados o divorciados, que son además las que mayor insatisfacción corporal presentan. Este parece ser un indicador de que el programa específico aplicado sobre imagen corporal provoca mayores mejoras sobre los sujetos que más lo necesitan, seguramente derivado de tener mayor margen de mejora. No obstante, se recuerda que estos resultados deben tomarse con moderación, puesto que la muestra en esta condición es muy pequeña $(N=7)$.

Los resultados de este estudio reflejan cómo sigue siendo relevante tener en cuenta la satisfacción corporal a la hora de plantear una intervención sobre un estado de ánimo depresivo o una baja autoestima en una persona mayor de 50 años, especialmente si es mujer, ya que la estabilidad de la influencia de la socialización dependiente del sexo parece extenderse a lo largo de todo el ciclo vital. Por último, se debe remarcar como principal limitación de este estudio la carencia de otros estudios similares que avalen los resultados obtenidos, cuestión que restringe la fortaleza de las conclusiones (Sánchez-Cabrero et al., 2019). 


\section{Referencias}

Abellán, A., Ayala, A., \& Pujol, R. (2017). Un perfil de las personas mayores en España, 2017. Indicadores estadísticos básicos (Informe. Envejecimiento en Red N. $\left.{ }^{\circ} 15\right)$. Madrid: Centro de Ciencias Humanas y Sociales, del Consejo Superior de Investigaciones Científicas. Recuperado de http://envejecimiento.csic.es/document os/documentos/enred-indicadoresbasicos 1 7.pdf

Baile, J. I., Guillén, F., \& Garrido, E. (2002). Insatisfacción corporal en adolescentes medida con el Body Shape Questionnaire (BSQ): efecto del anonimato, el sexo y la edad. Revista Internacional de Psicología Clínica y de la Salud, 2(3), 439-450. Recuperado de http://www.aepc.es/ijchp/ar ticulos_pdf/ijchp-49.pdf

Bailey, K. A., Gammage, K. L., \& van Ingen, C. (2019). Designing and implementing a positive body image program: Unchartered territory with a diverse team of participants. Action Research, 17(2), 146-161. https://doi .org/10.1177\%2F1476750318821169

Benton, C., \& Karazsia, B. T. (2015). The effect of thin and muscular images on women's body satisfaction. Body Image, 13, 22-27. htt ps://doi.org/10.1016/j.bodyim.2014.11.001

Bratt, C., Abrams, D., Swift, H. J., Vauclair, C. M., \& Marques, S. (2018). Perceived age discrimination across age in Europe: From an ageing society to a society for all ages. Developmental Psychology, 54(1), 167-180. h ttps://doi.org/10.1037/dev0000398

Cash, T. F. (1997). The body image workbook: An 8-step program for learning to like your looks. Oakland, CA: New Harbinger Publications.

Cash, T. F. (2015). Multidimensional BodySelf Relations Questionnaire (MBSRQ). En T. Wade (Ed.), Encyclopedia of feeding and eating disorders (pp. 1-4). Singapore: Springer. https://doi.org/10.1007/978-981287-087-2_3-1
Cash, T. F. (2017). Body image: A joyous journey. Body Image, 23, A1-A2. https:// doi.org/10.1016/j.bodyim.2017.11.001

Conti, M. A., Cordás, T. A., \& Latorre, M. R. D. O. (2009). A study of the validity and reliability of the Brazilian version of the Body Shape Questionnaire (BSQ) among adolescents. Revista Brasileira de Saúde Materno Infantil, 9(3), 331-338. https://doi. org/10.1590/s1519-38292009000300012

Cooper, P. J., Taylor, M. J., Cooper, Z., \& Fairburn, C. G. (1987). The development and validation of the Body Shape Questionnaire. International Journal of Eating Disorders, 6, 485-494. https://doi.org/10.1002/1098-108 x(198707)6:4\%3C485::aid-eat226006040 5\%3E3.0.co;2-o

Cundall, A., \& Guo, K. (2017). Women gaze behaviour in assessing female bodies: The effects of clothing, body size, own body composition and body satisfaction. Psychological Research, 81(1), 1-12. https://d oi.org/10.1007/s00426-015-0726-1

Fernández, J. G., González, I., Contreras, O., \& Cuevas, R. (2015). Relación entre imagen corporal y autoconcepto físico en mujeres adolescentes. Revista Latinoamericana de Psicología, 47(1), 25-33. https://doi.org/10.1 016/S0120-0534(15)30003-0

Garner, D. M., Olmstead, M. P., \& Polivy, J. (1983). Development and validation of a multidimensional eating disorder inventory for anorexia nervosa and bulimia. International Journal of Eating Disorders, 2(2), 15-34. https://doi.org/10.1037/t64315 $-000$

Grogan, S. (2016). Body image: Understanding body dissatisfaction in men, women and children. Londres: Routledge. https://doi.or g/10.4324/9781315681528

Gubrium, J. F., \& Holstein, J. A. (2006). The life course. En L. Reynolds \& N. HermanKinney (Eds.), Handbook of symbolic interactionism (pp. 835-855). Walnut Creek, CA: AltaMira Press.

Halliwell, E., Yager, Z., Paraskeva, N., Diedrichs, P. C., Smith, H., \& White, P. (2016). 
Body image in primary schools: A pilot evaluation of a primary school intervention program designed by teachers to improve children's body satisfaction. Body Image, 19, 133-141. https://doi.org/10.1016/j.bodyim. 2016.09.002

Homan, K. J., \& Tylka, T. L. (2018). Development and exploration of the gratitude model of body appreciation in women. Body Image, 25, 14-22. https://doi. org/10.1016/j.bodyim.2018.01.008

Hudson, N. W., Lucas, R. E., \& Donnellan, M. B. (2016). Getting older, feeling less? A crosssectional and longitudinal investigation of developmental patterns in experiential well-being. Psychology and Aging, 31(8), 847-861. https://doi.org/10.1037/pag00001 38

Instituto Nacional de Estadística. (2017). Índices de envejecimiento de la población española 2017. Madrid: Autor. Recuperado de http:/ /www.ine.es/jaxiT3/Datos.htm? $\mathrm{t}=1489$

Kihlstrom, J. F. (2015). What does the selflook like? En T. K. Srull \& R. S. Wyer, Jr. (Eds.), The mental representation of trait and autobiographical knowledge about the self (pp. 87-98). Nueva York: Psychology Press.

Kilpela, L. S., Blomquist, K., Verzijl, C., Wilfred, S., Beyl, R., \& Becker, C. B. (2016). The body project 4 all: A pilot randomized controlled trial of a mixed-gender dissonance-based body image program. International Journal of Eating Disorders, 49(6), 591-602. https://do i.org/10.1002/eat.22562

Kvalem, I. L., Træen, B., Markovic, A., \& von Soest, T. (2019). Body image development and sexual satisfaction: A prospective study from adolescence to adulthood. The Journal of Sex Research, 56(6), 791-801. https://doi. org/10.1080/00224499.2018.1518400

Kozar, J. M. (2004). Relationship of attitudes toward advertising images and self- perceptions of older women (Tesis doctoral). Dissertation Abstracts International Section A: Humanities and Social Sciences, 65(8-A), 3116. https://doi.o rg/10.31274/rtd-180813-14309
Longo, M. R. (2015). Implicit and explicit body representations. European Psychologist, 20(1), 6-15. https://doi.org/10.1177/09567 97610380700

Maganto, C., del Río, A., \& Roiz, O. (2002). Programa preventivo sobre Imagen corporal y Trastornos de la Alimentación (PICTA). Madrid: TEA.

Maganto, C., Garaigordobil, M., \& Kortabarria, L. (2017). Eating problems in adolescents and youths: Explanatory variables. The Spanish Journal of Psychology, 19(E81), 1-9. https://doi.org/10.1017/sjp.2016.74

Mangweth-Matzek, B., Rupp, C. I., Hausmann, A., Assmayr, K., Mariacher, E., Kemmler, G., ... \& Biebl, W. (2006). Never too old for eating disorders or body dissatisfaction: A community study of elderly women. International Journal of Eating Disorders, 39(7), 583-586. https://doi.org/10.1002/eat .20327

Markey, C. N., Markey, P. M., \& Birch, L. L. (2005). Interpersonal predictors of dieting practices among married couples. Journal of Family Psychology, 15, 464-475. https://doi. org/10.1037/0893-3200.15.3.464

McCabe, M. P., Connaughton, C., Tatangelo, G., Mellor, D., \& Busija, L. (2017). Healthy me: A gender-specific program to address body image concerns and risk factors among preadolescents. Body Image, 20, 20-30. http s://doi.org/10.1016/j.bodyim.2016.10.007

McDermott, E., Mullen, G., Moloney, J., Keegan, D., Byrne, K., Doherty, G. A., ... Mulcahy, H. E. (2015). Body image dissatisfaction: Clinical features, and psychosocial disability in inflammatory bowel disease. Inflammatory Bowel Diseases, 21 (2), 353-360. https://doi.org/10.1097/MI B.0000000000000287

McLean, S. A., Paxton, S. J., \& Wertheim, E. H. (2011). A body image and disordered eating intervention for women in midlife: A randomized controlled trial. Journal of Consulting and Clinical Psychology, 79(6), 751-758. https://doi.org/10.1037/a0026094 Mellor, D., Connaughton, C., McCabe, M. P., \& Tatangelo, G. (2017). Better with age: 
A health promotion program for men at midlife. Psychology of Men $\mathfrak{E}$ Masculinity, 18(1), 40-49. https://doi.org/10.1037/men0 000037

Raich, R. M., Mora, M., Soler, A., Ávila, C., Clos, I., \& Zapater, L. (1996). Adaptación de un instrumento de evaluación de la insatisfacción corporal (BSQ). Clínica y Salud, 7(1), 51- 66. Recuperado de https://journals.copmadrid.org/clysa/art/ f2217062e9a397a1dca429e7d70bc6ca

Rodgers, R. F., McLean, S. A., \& Paxton, S. J. (2015). Longitudinal relationships among internalization of the media ideal, peer social comparison, and body dissatisfaction: Implications for the tripartite influence model. Developmental Psychology, 51(5), 706-713. https://doi.org/10.1037/dev00000 13

Rosés-Gómez, M. del R. (2014). Desarrollo y evaluación de la eficacia de dos programas preventivos en comportamientos no saludables respecto al peso y la alimentación. Estudio piloto (Tesis doctoral inédita). Universitat Autònoma de Barcelona, Bellaterra. Recuperado de https://ddd.uab.cat/pub/tesi s/2014/hdl_10803_285161/mdrrg1de1.pdf

Sabik, N., \& Versey, H. S. (2016). Functional limitations, body perceptions, and health outcomes among older African American women. Cultural Diversity and Ethnic Minority Psychology, 22(4), 594-601. https:/ /doi.org/10.1037/cdp0000106

Sánchez-Cabrero, R. (2012). IMAGINA: programa de mejora de la autoestima y la imagen corporal para adultos. Logroño, ES: Siníndice.

Sánchez-Cabrero, R., Carranza, N., Novillo, M. A., \& Pericacho, F. J. (2019). The importance of physical appearance during the ageing process in Spain. Interrelation between body and life satisfaction during maturity and the old age. Activities, Adaptation $\mathcal{E}$ Aging, 43, 1-15. https://doi.or $\mathrm{g} / 10.1080 / 01924788.2019 .1651187$

Sánchez-Cabrero, R., \& Maganto, C. (2009). Satisfacción corporal y vital de personas mayores de 50 años en España. Revista de Psicología General y Aplicada, 62(4), 293-302. https://doi.org/10.30552/ejhr.v4i 2.105

Schoufour, J. D., de Jonge, E. A., Kiefte-de Jong, J. C., van Lenthe, F. J., Hofman, A., Nunn, S. P., \& Franco, O. H. (2018). Socio-economic indicators and diet quality in an older population. Maturitas, 107, 71-77. https://d oi.org/10.1016/j.maturitas.2017.10.010

Tylka, T. L., \& Wood-Barcalow, N. L. (2015). What is and what is not positive body image? Conceptual foundations and construct definition. Body Image, 14, 118-129. https://doi.org/10.1016/j.bodyim. 2015.04.001

Tylka, T. L., \& Homan, K. J. (2015). Exercise motives and positive body image in physically active college women and men: Exploring an expanded acceptance model of intuitive eating. Body Image, 15, 90-97. https://doi.org/10.1016/j.bodyim.20 15.07.003

Vega, A. V., Ramos, L. M., Barrios, M. L., \& Quintero, M. V. (2015). Imagen del cuerpo en adultos mayores. Salud en Movimiento, 7(1), 4-10. Recuperado de http://publicaciones.unisimonbolivar.ed u.co/rdigital/ojs/index.php/saludmov/articl e/view/1337

Voelker, D. K., Reel, J. J., \& Greenleaf, C. (2015). Weight status and body image perceptions in adolescents: Current perspectives. Adolescent Health, Medicine and Therapeutics, 6, 149-158. https://doi.or g/10.2147/AHMT.S68344

World Medical Association. (2013). WMA declaration of Helsinki: Ethical principles for medical research involving human subjects. Journal of the American Medical Association, 310(20), 2191-2194. https://d oi.org/10.1001/jama.2013.281053

Zeman, Z., \& Zeman, M. G. (2015). "Young" heart in "old" body. Experiences of ageing from older persons' perspective. Etnološka tribina: Godišnjak Hrvatskog etnološkog društva, 45(38), 72-85. https://doi.org/10.1 5378/1848-9540.2015.38.03 
MEJORA DE LA SATISFACCIÓN CORPORAL EN LA MADUREZ A TRAVÉS DE UN PROGRAMA ESPECÍFICO DE IMAGEN...

Notas

* Artículo de investigación. 